\title{
Exportación de nitrógeno y calcio mediante raleo en un rodal de Eucalyptus nitens de 5 años de edad, Chile
}

\author{
Export of nitrogen and calcium through the thinning of a five-year-old stand of \\ Eucalyptus nitens, Chile
}

\author{
Oscar Thiers Ea, Víctor Gerding ${ }^{a *}$, Juan E Schlatter ${ }^{a}$ \\ aUniversidad Austral de Chile, Facultad de Ciencias Forestales. \\ * Autor de correspondencia: aUniversidad Austral de Chile, Facultad de Ciencias Forestales, \\ casilla 567, Valdivia, Chile, tel.-fax: 56-63-221431, vgerding@uach.cl
}

\begin{abstract}
SUMMARY
The aim of the present paper was to assess the export of nitrogen and calcium in a five-year-old stand of Eucalyptus nitens $\left(41^{\circ} 01^{\prime} \mathrm{S}\right.$ y $73^{\circ} 27^{\prime}$ W; $200 \mathrm{~m}$ rise, Acrudoxic Hydric Hapludand red clay soil), with and without fertilization, due to the thinning with varying extents of biomass extraction (logs: only wood, trunks: wood plus bark, and whole tree). The plantation was established in 1996 and was fertilized $(\mathrm{F})$ with phosphoric rock before planting, in the spring of the same year (NPK + microelements), the next year (NPK) and the third year (NP). A similar surface (0.25 ha) was defined as the control plot (T). The thinning of 700 trees ha $^{-1}$ was $^{2}$ considered, leaving a residual density of 800 trees $\mathrm{ha}^{-1}$ with the pruning of the lowest third of the crown. Prior to the thinning, the above-ground biomass in $\mathrm{T}$ was $95.6 \mathrm{Mg} \mathrm{ha}^{-1}$ (accumulation of: $434 \mathrm{~kg} \mathrm{~N} \mathrm{ha}^{-1}$ and $306 \mathrm{~kg} \mathrm{Ca} \mathrm{ha}^{-1}$ ) and in $\mathrm{F}$ it was $121.3 \mathrm{Mg}$ $\mathrm{ha}^{-1}\left(516 \mathrm{~kg} \mathrm{~N} \mathrm{ha}^{-1}\right.$ and $\left.359 \mathrm{~kg} \mathrm{Ca} \mathrm{ha}^{-1}\right)$. Roots represented $6.2 \%$ and $5.4 \%$ of the total biomass, respectively. The extraction of $\operatorname{logs}$ (only wood) exported 16-18\% of the above-ground nitrogen and 5-6\% of the above-ground calcium in the stand, whereas the extraction of whole trees took out $41-44 \%$ of the nitrogen and calcium. The bark was the biomass component that accumulated the most calcium, reaching almost three times as much as that in the wood. Retention of the bark in the site can be significant for the supply of this element during rotation.
\end{abstract}

Key words: nutrients inventory, biomass, harvest waste, fertilization, intensive silviculture.

RESUMEN

El objetivo del presente trabajo fue evaluar la exportación de nitrógeno y calcio en un rodal de Eucalyptus nitens de 5 años de edad ( $41^{\circ} 01^{\prime}$ S y $73^{\circ} 27^{\prime}$ O; 200 m de elevación, suelo rojo arcilloso Acrudoxic Hydric Hapludand), con y sin fertilización, producto de raleo con diferentes grados de extracción de biomasa (fuste, tronco y árbol completo). La plantación se estableció en 1996 y se fertilizó (F) con roca fosfórica antes de plantar, en la primavera del mismo año (NPK + microelementos), al año siguiente (NPK) y al tercer año (NP). Una superficie semejante $\left(0,25\right.$ ha) fue definida como testigo (T). Se consideró el raleo de 700 árboles ha ${ }^{-1}$, dejando una densidad residual de 800 árboles ha ${ }^{-1}$ con poda del tercio inferior de la copa. Previo al raleo la biomasa aérea en T fue de 95,6 $\mathrm{Mg} \mathrm{ha}^{-1}$ (acumulación de: $434 \mathrm{~kg} \mathrm{~N} \mathrm{ha}^{-1}$ y $306 \mathrm{~kg} \mathrm{Ca} \mathrm{ha}^{-1}$ ) y en F de 121,3 $\mathrm{Mg} \mathrm{ha}^{-1}\left(516 \mathrm{~kg} \mathrm{~N} \mathrm{ha}^{-1}\right.$ y $359 \mathrm{~kg} \mathrm{Ca} \mathrm{ha}^{-1}$ ). Las raíces representaron 6,2\% y 5,4\% de la biomasa total, respectivamente. La extracción del fuste (sólo madera) exportó 16-18\% del nitrógeno y 5-6\% de calcio aéreo del rodal, mientras que la extracción del árbol completo extrajo 41-44\% del nitrógeno y del calcio. La corteza fue el componente de biomasa que más calcio acumuló, alcanzando a casi el triple que en la madera. La retención de la corteza en el sitio puede ser significativa para el abastecimiento de este elemento durante la rotación.

Palabras clave: inventario nutritivo, biomasa aérea, desechos de cosecha, fertilización, silvicultura intensiva.

\section{INTRODUCCIÓN}

La superficie total de Eucalyptus nitens (Deane et Maiden) Maiden en Chile alcanza a 150.000 ha, existiendo aún cerca de 600.000 ha potenciales para su establecimiento en el sur del país (INFOR 2004). El establecimiento de plantaciones de E. nitens en esta región responde fundamentalmente a su rápido crecimiento, su resistencia a bajas temperaturas y la cercanía a centros de consumo industrial.

En las plantaciones de rápido crecimiento la silvicultura intensiva es clave para mantener o aumentar su productividad en el futuro (Fox 2000, Goncalves et al. 2004). Producto de la intensificación de la silvicultura han aumentado las tasas de crecimiento y con ello se ha incrementado la demanda nutritiva en períodos más cortos. La mantención 
de la fertilidad del suelo debe considerar, entre otros, el manejo nutritivo que incluye aplicación de fertilizantes y manejo de los residuos de cosecha (Yost et al. 1988, Smethurst et al. 2001, Yamada et al. 2004, Ringrose y Neilsen 2005). Por sus altas tasas de crecimiento inicial, E. nitens presenta una gran demanda de nutrientes que, en general, no alcanza a ser cubierta por la disponibilidad en el suelo y las propias reservas de las plantas. Por ello el déficit debe ser suplido con fertilizantes (Bonomelli y Suárez 1999, Suárez y Bonomelli 2001, Bonomelli et al. 2002, Geldres 2005ab).

Los efectos en el balance de nutrientes de diferentes métodos e intensidades de extracción de biomasa en rodales de E. nitens han sido poco analizados en Chile (Yamada et al. 2004, Geldres 2005ab). En otras regiones del mundo se han cuantificado en plantaciones de Eucalyptus spp. la acumulación y la extracción de elementos nutritivos a través de la biomasa cosechada (Madgwick et al. 1981, Frederick et al. 1985, Misra et al. 1998b, Laclau et al. 2001, Schumacher y Caldeira 2001, Muñoz 2002, Sankaran et al. 2005); entre los elementos con mayores niveles de exportación se identifican al nitrógeno y al calcio. Las exportaciones de elementos nutritivos dependen de la cantidad de biomasa cosechada, la cual varía según el tipo de cosecha practicada (árbol completo, troncos con corteza o descortezados) y también de la edad de rotación o la edad a la cual se ralea (Raison y Crane 1986).

El objetivo del presente trabajo fue evaluar la exportación de nitrógeno y calcio en un rodal de Eucalyptus nitens de 5 años de edad, con y sin fertilización, producto de raleo con diferentes grados de extracción de biomasa (fuste, tronco y árbol completo).

\section{MÉTODOS}

Establecimiento de la plantación y tratamiento de fertilización. La plantación de E. nitens se ubicó en el predio Pichimaule, comuna de Fresia (41 $01^{\prime}$ 'S y $73^{\circ} 27^{\prime}$ O; 200 m s.n.m.). El clima es de costa occidental con influencia mediterránea, donde la precipitación anual alcanza 1.600$2.000 \mathrm{~mm}$ y presenta un periodo seco de 1-2 meses al año. El suelo es del tipo rojo arcilloso de la serie Crucero (Acrudoxic Hydric Hapludand) (CIREN 2001). Este suelo es profundo, de textura franca arcillosa, con buena estructura y drenaje interno moderado; presenta alta acidez y baja disponibilidad de fósforo (Geldres 2005ab, Geldres et al. 2006). El uso anterior correspondió a pradera natural, con antecedentes agrícolas.

La preparación del terreno, en marzo de 1996, consideró control químico de malezas en toda la superficie, en mayo del mismo año se subsoló a $50 \mathrm{~cm}$ de profundidad en las líneas de plantación. La plantación se estableció en junio ( $4 \mathrm{~m} \times 1,5 \mathrm{~m})$, con plantas producidas en contenedores con semilla de Macalyster (Victoria Central, Australia) y se seleccionaron por altura $(25-30 \mathrm{~cm})$ y diámetro de cuello (4 mm). En octubre de 1996 se hizo un nuevo control químico de malezas y posteriormente en 1997 y 1998 controles manuales. Estas condiciones generales del establecimiento constituyeron el tratamiento testigo $(\mathrm{T})$. El tratamiento de fertilización (F) consideró la aplicación de roca fosfórica (Bifox) equivalente a 49 $\mathrm{kg} \mathrm{ha}^{-1}$ de fósforo en las líneas de plantación, durante la preparación del suelo. Posteriormente, en octubre de 1996, se fertilizó con $135 \mathrm{~g}_{\text {planta }}{ }^{-1}$ de NPK (20:25:5), incorporados en dos bandas de $40 \mathrm{~cm}$ de largo y $5 \mathrm{~cm}$ de profundidad, a $5-10 \mathrm{~cm}$ de las plantas. Esta fertilización correspondió a $36 \mathrm{~kg} \mathrm{ha}^{-1}$ de nitrógeno (urea), 20 de fósforo (superfosfato triple) y 5,6 de potasio (sulfato de potasio); la mezcla incluyó los micronutrientes zinc, cobre (sulfatos) y boro (boronatrocalcita). En septiembre de 1997 se fertilizó al voleo en fajas, junto a las hileras de árboles, con $45 \mathrm{~kg} \mathrm{ha}^{-1}$ de nitrógeno (urea), $48 \mathrm{de}$ fósforo (superfosfato triple), 10 de potasio (muriato), 4,8 de boro (boronatrocalcita), 1,25 de cobre (sulfato) y 1,15 de zinc (sulfato). En agosto de 1999 se fertilizó al voleo en toda la superficie del rodal con $230 \mathrm{~kg} \mathrm{ha}^{-1}$ de nitrógeno (supernitro) y 100 de fósforo (superfosfato triple). Cada tratamiento (T y F) abarcó una superficie de 0,25 ha $(50 \times 50 \mathrm{~m})$. Las dosis aplicadas en las fertilizaciones se basaron en el modelo racional que considera la oferta del suelo, la demanda de la especie a una edad determinada y la eficiencia del producto (Rodríguez 1993, Álvarez et al. 1999). La oferta nutritiva disponible del suelo (0-20 $\mathrm{cm}$ y para una arraigabilidad máxima estimada del 50\%) fue de $314 \mathrm{~kg} \mathrm{Ca} \mathrm{ha}^{-1}, 104 \mathrm{~kg} \mathrm{~K} \mathrm{ha}^{-1}, 4 \mathrm{~kg} \mathrm{P} \mathrm{ha}^{-1}$ y de $30 \mathrm{~kg} \mathrm{~N} \mathrm{ha}^{-1}$; esta última estimada a partir del contenido de nitrógeno total del suelo y una tasa de mineralización de $1 \%$ anual (Geldres 2005ab).

Biomasa, nitrógeno y calcio. Al año 5 de la plantación (2001) se realizó un inventario forestal donde se obtuvo el diámetro a 1,3 m (DAP), la altura total y la densidad de los rodales. Se determinó la biomasa total (aérea y radicular), a través de la cosecha de árboles representativos, de acuerdo con la distribución diamétrica. Los componentes de la copa se separaron según su ubicación vertical ( $1 / 3$ superior, $1 / 3$ medio y $1 / 3$ inferior), separando hojas (juveniles y adultas), ramas vivas (gruesas $\varnothing>1$ $\mathrm{cm}$ y finas $\emptyset \leq 1 \mathrm{~cm}$ ) y ramas muertas. Desde la base del tronco y cada $2 \mathrm{~m}$ se obtuvieron muestras (rodelas) para los análisis químicos y la determinación de la proporción madera/corteza. Las raíces se muestrearon en seis columnas de suelo de $10 \times 10 \mathrm{~cm}$ de superficie y $110 \mathrm{~cm}$ de profundidad, separándolas por diámetro $(\varnothing>3 \mathrm{~cm}, 0,2$ a $3 \mathrm{~cm}$ y $<0,2 \mathrm{~cm}$ ). Las raíces principales se obtuvieron de dos árboles de diámetro medio cuadrático (DMC) en cada tratamiento (Geldres 2005a). Para todos los componentes de la biomasa se determinó peso húmedo en terreno y, en laboratorio, peso seco (contenidos de humedad) y contenidos de nitrógeno (Kjeldahl) y calcio (espectrofotometría de absorción atómica). 
Utilizando la información de distribución diamétrica, obtenida de cuatro unidades muestrales $(12,5 \times 50 \mathrm{~m})$ en que se dividió la superficie de cada tratamiento, y con las funciones de biomasa de Minte (2004), se estimó la biomasa total aérea de los rodales. En este último aspecto la estimación de la biomasa difiere de aquella realizada por Geldres et al. (2006) en este mismo ensayo. La biomasa se distribuyó en sus distintos componentes según su porcentaje de participación en el total (Geldres 2005a, Geldres et al. 2006). Con los contenidos de nitrógeno y calcio se calcularon las cantidades de estos elementos en cada componente de la biomasa. El análisis de los datos de biomasa y cantidades de elementos nutritivos se hizo sobre la base de la superficie total de cada tratamiento. Además se calculó la eficiencia $\left(\mathrm{kg} \mathrm{kg}^{-1}\right)$, que corresponde a la cantidad de biomasa producida por unidad de elemento nutritivo acumulado (Raison y Crane 1986). Las comparaciones entre tratamientos se hicieron a través de prueba t.

Características de los rodales y supuestos para la intervención silvicultural. La densidad inicial de ambos rodales fue de 1.500 árboles ha ${ }^{-1}$. A los 5 años de edad, previo al raleo, en el testigo el DMC fue de $15,1 \mathrm{~cm}$ $\mathrm{y}$ en el fertilizado de $16,1 \mathrm{~cm}$; las respectivas alturas totales fueron de $14 \mathrm{~m}$ y $16,3 \mathrm{~m}$. El rodal fertilizado presentó más árboles de diámetros superiores (DAP $\geq$ $14 \mathrm{~cm}$ ) y, además, alturas totales mayores que en el rodal testigo en cada clase diamétrica. A partir de esta situación se simuló, a la edad de 5 años, un raleo de 700 árboles $\mathrm{ha}^{-1}$, variando levemente con respecto a la corta intermedia que se realizó en los rodales de estudio. La simulación incluyó la totalidad de las clases diamétricas, pero se concentró más fuertemente en las clases menores, equivalente a un raleo por lo bajo de intensidad media a alta (Nyland 2002) (figura 1).

Para el raleo se consideraron tres tipos de extracción: árbol completo (biomasa aérea), troncos (madera con corteza) y fustes (sólo madera); en estos dos últimos casos los desechos del raleo permanecen sobre el suelo. También se simuló una poda del tercio inferior de la copa de los 800 árboles ha $^{-1}$ residuales, dejando los desechos esparcidos sobre el suelo.

\section{RESULTADOS}

La biomasa aérea fue de 95,6 $\mathrm{Mg} \mathrm{ha}^{-1}$ en el rodal testigo y de $121,3 \mathrm{Mg} \mathrm{ha}^{-1}$ en el fertilizado (cuadro 1). Con la fertilización se obtuvo $27 \%$ más de biomasa aérea y $9 \%$ más de biomasa radicular. Los árboles raleados fueron equivalentes al $41 \%$ de la biomasa aérea en el testigo y al $44 \%$ en el fertilizado. En la biomasa aérea del rodal fertilizado se acumuló $19 \%$ más nitrógeno y $17 \%$ más de calcio con respecto al rodal testigo (cuadro 1). La eficiencia en la producción de biomasa total, obtenida a partir del cuadro 1, fue similar en ambos tratamientos, tanto para nitrógeno (214-222 $\mathrm{kg} \mathrm{kg}^{-1}$ ) como para calcio (311-322 $\mathrm{kg} \mathrm{kg}^{-1}$ ), con una leve ventaja de $4 \%$ del fertilizado sobre el testigo. Considerando sólo la biomasa aérea, la eficiencia fue algo mayor para el rodal fertilizado con respecto al testigo (incremento de $7 \%$ para nitrógeno y de $8 \%$ para calcio).

El rodal fertilizado acumuló en las raíces $50 \%$ más de nitrógeno y $27 \%$ más de calcio con respecto al testigo. La poda en el testigo significó un aporte al mantillo de $77 \mathrm{~kg} \mathrm{ha}^{-1}$ de nitrógeno y $63 \mathrm{~kg} \mathrm{ha}^{-1}$ de calcio; en el fertilizado fue de 95 y $86 \mathrm{~kg} \mathrm{ha}^{-1}$, respectivamente. Por tanto, existió una mayor devolución hacia el mantillo en el rodal fertilizado, con respecto al testigo, de $23 \%$ de nitrógeno y $37 \%$ de calcio.

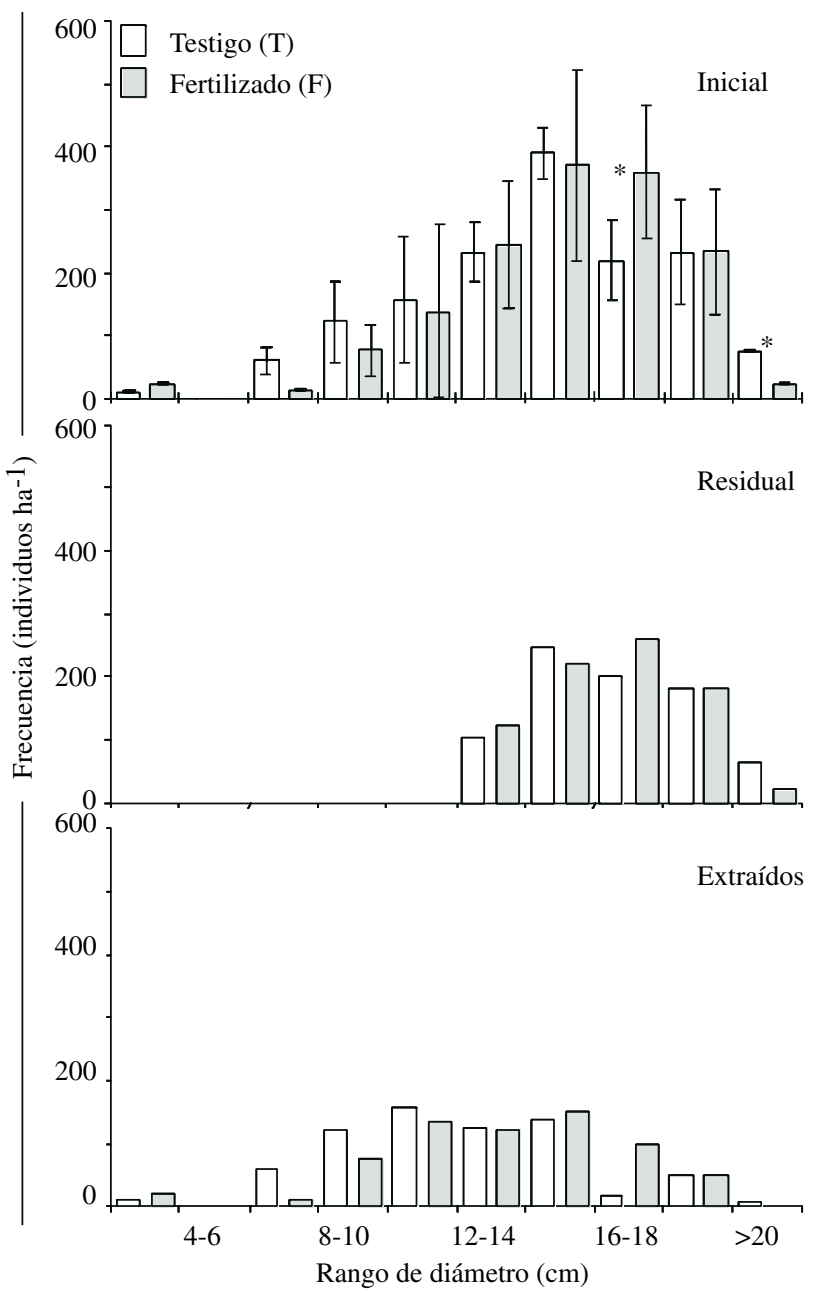

Figura 1. Distribución diamétrica de los rodales testigo (T) y fertilizado (F) antes del raleo (Inicial), después del raleo (Residual) y de los árboles extraídos (Extraídos). Líneas verticales sobre las columnas corresponden a la desviación estándar. *Diferencia significativa entre rodales $(P<0,05)$.

Diameter distribution in the control (T) and fertilized (F) stands before thinning (Inicial), after thinning (Residual) and of the removed trees (Extraídos). The vertical lines on the columns correspond to the standard deviation. *Significative difference between stands $(P<0.05)$. 
Cuadro 1. Biomasa, nitrógeno y calcio por componente para los tratamientos testigo $(\mathrm{T})$ y con fertilización (F), considerando prey posraleo.

Biomass, nitrogen and calcium in the components for control $(\mathrm{T})$ and fertilized $(\mathrm{F})$ treatments, before and after thinning.

\begin{tabular}{|c|c|c|c|c|c|c|c|c|c|c|c|c|}
\hline & \multicolumn{3}{|c|}{ Biomasa $\left(\mathrm{kg} \mathrm{ha}^{-1}\right)$} & \multirow[b]{2}{*}{$\%^{2}$} & \multicolumn{3}{|c|}{ Nitrógeno $\left(\mathrm{kg} \mathrm{ha}^{-1}\right)$} & \multirow[b]{2}{*}{$\%^{3}$} & \multicolumn{3}{|c|}{ Calcio $\left(\mathrm{kg} \mathrm{ha}^{-1}\right)$} & \multirow[b]{2}{*}{$\%^{3}$} \\
\hline & Inicial & Residual & Extraída $^{1}$ & & Inicial & Residual & Extraída ${ }^{1}$ & & Inicial & Residual & Extraída ${ }^{1}$ & \\
\hline Componente & \multicolumn{12}{|c|}{ Testigo } \\
\hline Hojas & 12.260 & 7.262 & 4.998 & 5 & 166 & 98 & 67 & 16 & 82 & 49 & 33 & 11 \\
\hline Ramas & 17.283 & 10.237 & 7.046 & 7 & 65 & 38 & 26 & 6 & 91 & 54 & 37 & 12 \\
\hline Corteza & 7.210 & 4.270 & 2.939 & 3 & 37 & 22 & 15 & 3 & 98 & 58 & 40 & 13 \\
\hline Madera & 58.795 & 34.825 & 23.970 & 25 & 167 & 99 & 68 & 16 & 35 & 21 & 14 & 5 \\
\hline Subtotal aéreo & 95.547 & 56.593 & 38.954 & 41 & 434 & 257 & 177 & 41 & 306 & 181 & 125 & 41 \\
\hline Raíces & 6.325 & 3.746 & 2.579 & & 42 & 25 & 17 & & 22 & 13 & 9 & \\
\hline Total & 101.872 & 60.340 & 41.532 & & 476 & 282 & 194 & & 328 & 194 & 134 & \\
\hline Componente & \multicolumn{12}{|c|}{ Fertilizado } \\
\hline Hojas & 14.954 & 8.310 & 6.643 & 5 & 185 & 103 & 82 & 16 & 101 & 56 & 45 & 13 \\
\hline Ramas & 20.828 & 11.575 & 9.253 & 8 & 76 & 42 & 34 & 7 & 95 & 53 & 42 & 12 \\
\hline Corteza & 9.036 & 5.022 & 4.014 & 3 & 51 & 29 & 23 & 4 & 118 & 65 & 52 & 15 \\
\hline Madera & 76.524 & 42.528 & 33.997 & 28 & 205 & 114 & 91 & 18 & 46 & 25 & 20 & 6 \\
\hline Subtotal aéreo & 121.342 & 67.435 & 53.907 & 44 & 516 & 287 & 229 & 44 & 359 & 200 & 160 & 44 \\
\hline Raíces & 6.916 & 3.844 & 3.073 & & 63 & 35 & 28 & & 38 & 21 & 17 & \\
\hline Total & 128.258 & 71.278 & 56.980 & & 579 & 322 & 257 & & 398 & 221 & 177 & \\
\hline
\end{tabular}

${ }^{1}$ Cantidad extraída por raleo. ${ }^{2}$ Porcentaje de biomasa extraída por raleo respecto del subtotal inicial de biomasa aérea. ${ }^{3}$ Porcentaje de la cantidad de elemento extraído por raleo respecto del subtotal inicial del mismo elemento en la biomasa aérea.

El nitrógeno acumulado en la biomasa total, como promedio de los dos tratamientos (cuadro 1), se concentró en las hojas y en la madera con una proporción aproximada de $35 \%$ en cada uno de estos componentes. Sin embargo, las hojas representaron sólo el $12 \%$ y la madera el $59 \%$ de la biomasa total en cada rodal. El calcio, en cambio, mostró una baja acumulación en la madera (11\% del total) pero su participación fue alta en la corteza (30\% del total), considerando que este último componente representó sólo el 7\% de la biomasa total. Las hojas y las ramas acumularon un poco menos calcio que la corteza, no obstante que presentaron mayor cantidad de biomasa que la corteza (1,7 y 2,4 veces, respectivamente).

En comparación con la extracción de fustes, que fue el tipo de cosecha más conservador, la exportación de troncos, que incluyó la corteza, significó un aumento de la pérdida de nitrógeno de 22-25\%; pero casi triplicó $(2,6-$ 2,8 veces) la salida de calcio del sitio. Por su parte, la cosecha de árbol completo significó un mayor aumento de las exportaciones de nitrógeno (1,5-1,6 veces) y de calcio (6,9-7,7 veces) (cuadro 2).

En el rodal fertilizado la exportación nutritiva fue $20-30 \%$ mayor que en el testigo. La extracción del fuste (sólo madera) exportó 16-18\% del nitrógeno aéreo acumulado y un 5-6\% del calcio aéreo; la cosecha de árbol completo extrajo 41-44\% del nitrógeno y del calcio aéreo del rodal; y la extracción del tronco (madera y corteza) en ambos tratamientos alcanzó niveles intermedios, similares para los dos elementos nutritivos, exportando $18-22 \%$ de lo acumulado en la biomasa aérea (cuadro 2).

\section{DISCUSIÓN}

Las cantidades de nitrógeno y calcio acumuladas en los distintos compartimentos de la biomasa aérea de este estudio son coincidentes con lo reportado por otros autores con respecto a plantaciones de Eucalyptus spp. (Madgwick et al. 1981, Frederick et al. 1985, Yost et al. 1988, Misra et al. 1998b, Laclau et al. 2001, Schumacher y Caldeira 2001, Muñoz 2002, Sankaran et al. 2005). Se destaca la alta proporción de nitrógeno en las hojas y en la madera, que presentan similares cantidades, alcanzando en conjunto aproximadamente el $70 \%$ del total acumulado en la biomasa. Por el contrario, el calcio presenta baja acumulación en la madera y mayores cantidades en la corteza, las ramas y las 
Cuadro 2. Biomasa, nitrógeno y calcio exportados según tipo de cosecha en el raleo para los tratamientos testigo (T) y con fertilización $(\mathrm{F})$.

Biomass, nitrogen and calcium export intensity through thinning in the control $(\mathrm{T})$ and fertilized $(\mathrm{F})$ treatments.

\begin{tabular}{|c|c|c|c|c|c|c|c|c|c|c|c|c|}
\hline & \multicolumn{3}{|c|}{ Biomasa $\left(\mathrm{kg} \mathrm{ha}^{-1}\right)$} & \multirow[b]{2}{*}{$\%^{1}$} & \multicolumn{3}{|c|}{ Nitrógeno $\left(\mathrm{kg} \mathrm{ha}^{-1}\right)$} & \multirow[b]{2}{*}{$\%^{2}$} & \multicolumn{3}{|c|}{ Calcio $\left(\mathrm{kg} \mathrm{ha}^{-1}\right)$} & \multirow[b]{2}{*}{$\%^{2}$} \\
\hline & Inicial & Residual & Extraída & & Inicial & Residual & Extraída & & Inicial & Residual & Extraída & \\
\hline Tipo de cosecha & \multicolumn{12}{|c|}{ Testigo } \\
\hline Fuste $^{3}$ & 58.795 & 34.825 & 23.970 & 25 & 167 & 99 & 68 & 16 & 35 & 21 & 14 & 5 \\
\hline Tronco $^{4}$ & 66.004 & 39.095 & 26.909 & 28 & 204 & 121 & 83 & 19 & 133 & 79 & 54 & 18 \\
\hline Completo $^{5}$ & 95.547 & 56.593 & 38.954 & 41 & 434 & 257 & 177 & 41 & 306 & 181 & 125 & 41 \\
\hline Tipo de cosecha & \multicolumn{12}{|c|}{ Fertilizado } \\
\hline Fuste $^{3}$ & 76.524 & 42.528 & 33.997 & 28 & 205 & 114 & 91 & 18 & 46 & 25 & 20 & 6 \\
\hline Tronco $^{4}$ & 85.560 & 47.549 & 38.011 & 31 & 256 & 142 & 114 & 22 & 163 & 91 & 73 & 20 \\
\hline Completo $^{5}$ & 121.342 & 67.437 & 53.907 & 44 & 516 & 287 & 229 & 44 & 359 & 200 & 160 & 44 \\
\hline
\end{tabular}

${ }^{1}$ Porcentaje de biomasa extraída respecto de la biomasa total aérea inicial (árbol completo). ${ }^{2}$ Porcentaje de la cantidad de elemento extraído por raleo respecto del total acumulado. ${ }^{3}$ Sólo madera. ${ }^{4}$ Madera y corteza. ${ }^{5}$ Madera, corteza, ramas y hojas.

hojas. En cada uno de estos últimos tres compartimentos el calcio representa aproximadamente un $30 \%$ del total acumulado en la biomasa. Los niveles de eficiencia para ambos elementos en la producción de biomasa fueron menores a los determinados por otros autores en Eucalyptus spp., especialmente para nitrógeno (Santana et al. 2002, Stape et al. 2004). La existencia de diferencias mínimas en eficiencia (4-8\%) entre tratamientos podría explicarse por la calidad del sitio media a alta (Geldres 2005ab), pues no existen restricciones climáticas y las restricciones edáficas son sólo marginales (niveles bajos de fósforo). La ausencia de factores limitantes marcados, especialmente nutricionales, podría condicionar la expresión del manejo nutritivo (Fox 2000).

Sin embargo, el efecto de la fertilización sobre la biomasa se aprecia de mayor magnitud en los componentes aéreos que en el radicular, coincidiendo con la tendencia observada para esta misma especie por Misra et al. (1998a). La biomasa aérea del rodal fertilizado fue un $26 \%$ mayor con respecto al testigo mientras que la radicular lo superó sólo en 9\%. Sin embargo, la distribución de nitrógeno y calcio prácticamente no se modificó por efecto de la fertilización (cuadro 1).

La poda al quinto año representa una devolución de elementos al mantillo que equivale a 2-3 veces la disponibilidad de nitrógeno y de 20-25\% del calcio disponible del suelo superficial $(0-20 \mathrm{~cm})$. De acuerdo con estos resultados, la poda puede ser de mayor efecto en el abastecimiento de nitrógeno que de calcio a través de la descomposición y mineralización de los desechos. Según Laclau et al. (2003), el abastecimiento de calcio en Eucalyptus spp. es muy dependiente de la oferta del suelo.
El tipo de cosecha puede incidir sobre el balance nutritivo del ecosistema de una plantación según la extracción de biomasa que ocasione, la cual condicionará la exportación de elementos nutritivos. Tanto para el nitrógeno como para el calcio la cosecha de árbol completo ocasiona naturalmente la mayor pérdida de tales elementos del sitio. En este estudio, la exportación de nitrógeno con el raleo, en el rodal testigo, puede ser dos a seis veces la cantidad disponible anual de este elemento en el suelo superficial según el tipo de cosecha aplicado. Para el rodal fertilizado estas proporciones son mayores, de tres a ocho veces. En todo caso, el suelo bajo estas plantaciones presenta una alta reserva de nitrógeno total que otorga estabilidad al sistema en el largo plazo (Gerding y Schlatter 1999).

La exportación de calcio es menor que la de nitrógeno (cuadro 2) y además el suelo presenta mayor oferta de calcio disponible. Con el raleo, en el escenario de mayor extracción de biomasa, la exportación de calcio alcanza el 50\% de la disponibilidad anual en el suelo superficial. Sin embargo, Gerding y Schlatter (1999) determinaron que suelos rojo arcillosos con plantaciones de Pinus radiata D. Don en Chile presentan una condición de inestabilidad con respecto a este elemento en el largo plazo. Esto puede significar una condición de mayor restricción en plantaciones de E. nitens debido a su mayor demanda por calcio en comparación con $P$. radiata (Schlatter et al. 1998, Ouro et al. 2001).

La exportación de corteza con los troncos incrementa significativamente las pérdidas de calcio, pero ejerce menor efecto en las salidas de nitrógeno. Estos incrementos en la exportación de elementos, por sobre la proporción 
de biomasa involucrada, se deben a los contenidos de nitrógeno y calcio en los distintos tejidos. El alto contenido de calcio en la corteza observado es coincidente con lo expresado en la literatura para Eucalyptus spp. (Madgwick et al. 1981, Frederick et al. 1985, Goya et al. 1997, Muñoz 2002, Bonomelli et al. 2002, Sankaran et al. 2005).

Dentro de los fundamentos para mantener la productividad del sitio en el largo plazo está el manejo nutritivo del suelo (Fox 2000, Smethurst et al. 2001, Ringrose y Neilsen 2005). Ello incluye los principios de minimizar la exportación de biomasa, manejar adecuadamente los desechos de la cosecha y reponer los elementos nutritivos extraídos por la cosecha, entre otros (Gerding 1991, Hunter 2001). De acuerdo con los resultados de este estudio, coincidiendo con otros autores (Madgwick et al. 1981, Frederick et al. 1985, Yost et al. 1988, Laclau et al. 2001, Schumacher y Caldeira 2001, Sankaran et al. 2005), para minimizar las pérdidas de elementos nutritivos y materia orgánica, tanto los raleos como la cosecha de plantaciones de rotaciones cortas deberían considerar sólo la extracción de fustes, evitando la exportación de corteza y copas de los árboles.

En consecuencia, tanto en los raleos como en la cosecha final, el desramado y descortezado en el mismo sitio de volteo serían prácticas de manejo adecuadas para minimizar los niveles de extracción de biomasa y de elementos nutritivos. En el caso de una faena de descortezado en un punto del rodal, que concentra los desechos en una pequeña área, el manejo poscosecha de tales desechos debiera considerar su redistribución homogénea sobre el suelo del rodal, evitando una excesiva concentración de biomasa en algunos sectores y su pérdida en otros. También está la posibilidad de incorporar los desechos al suelo superficial (Jones et al. 1999), lo que requiere de un análisis específico del caso según las características edáficas y climáticas del sitio.

No obstante lo anterior, una práctica habitual de cosecha en plantaciones forestales en Chile, inadecuada para el mantenimiento de la productividad, es la de remover los árboles completos para desramarlos en algunas áreas pequeñas de procesamiento y carguío. Con ello se genera una acumulación de desechos en tales áreas y, en consecuencia, se produce una cosecha de árbol completo en la mayor parte de la superficie del rodal. Además, generalmente no se descorteza en el mismo rodal, sino que se realiza esto en las industrias que consumen la madera como materia prima, aumentando las pérdidas de materia orgánica y elementos nutritivos del sitio.

Una silvicultura intensiva, en particular con respecto al balance nutritivo, debe considerar además los costos financieros involucrados con una visión de largo plazo, con el fin de aumentar o mantener la rentabilidad en cada caso. Esto requiere un análisis costo/beneficio proyectado a varias rotaciones (Hagner 1995, Mackensen y Fölster 2000). Entre los principales costos a considerar están los de cosecha, del manejo posterior de los desechos, del establecimiento de la nueva plantación y de las fertilizaciones que debieran ser realizadas.

\section{AGRADECIMIENTOS}

Los autores agradecen el apoyo a la realización del presente trabajo a los proyectos FONDECYT 1010174 y DID-S-200024 de la Universidad Austral de Chile.

\section{REFERENCIAS}

Álvarez J, J Rodríguez, D Suárez. 1999. Mejoramiento de la productividad de plantaciones de Pinus radiata D. Don, a través de un modelo racional de fertilización. Bosque 20(1): 23-36.

Bonomelli C, D Suárez. 1999. Fertilización del eucalipto. 1. Efecto sobre la acumulación de biomasa. Ciencia e Investigación Agraria 26: 1-10.

Bonomelli C, I Peña, D Suárez. 2002. Contribución de la hojas en el proceso de reciclaje de $\mathrm{N}$ y $\mathrm{P}$ en Eucalyptus $s p$. Bosque 23(1): 61-77.

CIREN (Centro de Información de Recursos Naturales, CL). 2001. Estudio agrológico X Región. Descripciones de suelos, materiales y símbolos. Tomo I. Santiago, Chile. Centro de Investigación de Recursos Naturales. 199 p.

Fox T. 2000. Sustained productivity in intensively managed forest plantations. Forest Ecology and Management 138: 187-202.

Frederick DJ, HAI Madgwick, MF Jurguensen, GR Oliver. 1985. Dry matter content and nutrient distribution in an age series of Eucalyptus regnans plantations in New Zealand. New Zealand Journal of Forestry Science 15 (2): 158-179.

Geldres E. 2005a. Exportación de nitrógeno y potasio con la cosecha de Eucalyptus nitens. Tesis Magíster en Gestión Ambiental. Valdivia, Chile. Facultad de Ciencias Forestales, Universidad Austral de Chile. 45 p.

Geldres E. 2005b. Dinámica de fósforo y calcio en Eucalyptus nitens de 4 a 7 años en un suelo rojo arcilloso, X Región. Tesis Magíster en Ciencias. Valdivia, Chile. Facultad de Ciencias Forestales, Universidad Austral de Chile. 89 p.

Geldres E, V Gerding, JE Schlatter. 2006. Biomasa de Eucalyptus nitens de 4-7 años de edad en un rodal de la X Región, Chile. Bosque 27(3): 223-230.

Gerding V. 1991. Pinus radiata-Plantagen in Zentralchile: Standortfaktoren der Produktivität und Nährstoffverteilung in Beständen. Tesis Dr. Göttingen, Alemania. Forstwissenschftlichen Fachbereichs. Universidad Georg August-Göttingen. $182 \mathrm{p}$.

Gerding V, JE Schlatter. 1999. Estabilidad nutritiva de plantaciones de Pinus radiata D. Don en cinco sitios característicos de la VIII Región. Bosque 20(2): 107-115.

Goncalves JL, JL Stape, JP Laclau, P Smethurst, JL Gava. 2004. Silvicultural effects on the productivity and wood quality of eucalypt plantations. Forest Ecology and Management 193 (2004): 45-61.

Goya J, J Frangi, F Dalla Tea, MA Marco, F Larocca. 1997. Biomasa, productividad y contenido de nutrientes en plantaciones de Eucalyptus grandis en el NE de la provincia 
de Entre Ríos. In XVII Jornadas Forestales de Entre Ríos - Concordia, Argentina. p. III 1-19.

Hagner S. 1995. Silviculture in boreal forests. Unasylva 46(181): $18-25$.

Hunter I. 2001. Above ground biomass and nutrient uptake of three tree species (Eucalyptus camaldulensis, Eucalyptus grandis and Dalbergia sissoo) as affected by irrigation and fertiliser, at 3 years of age, in southern India. Forest Ecology and Management 144: 189-199.

INFOR (Instituto Forestal, CL). 2004. Eucalyptus nitens en Chile: Primera monografía. Santiago. Chile. Instituto Forestal. (Informe Técnico $\mathrm{N}^{\circ} 165$ ). $143 \mathrm{p}$.

Jones HE, M Madeira, L Herraez, J Dighton, A Fabiâo, F González-Rio, M Fernandez Marcos, C Gomez, M Tomé, H Feith, MC Magalhâes, G Howson. 1999. The effect of organic-matter management on the productivity of Eucalyptus globulus stands in Spain and Portugal: tree growth and harvest residue decomposition in relation to site and treatment. Forest Ecology and Management 122: 73-86.

Laclau JP, JP Bouillet, J Ranger, R Joffre, R Gouma, A Saya. 2001. Dynamics of Nutrient Translocation in Stemwood across an Age Series of a Eucalyptus Hybrid. Annals of Botany 88: 1079-1092.

Laclau JP, P Deleporte, J Ranger, JP Bouillet, G Kazotti. 2003. Nutrient Dynamics throughout the Rotation of Eucalyptus Clonal Stand in Congo. Annals of Botany 91: 879-892.

Mackensen J, H Fölster. 2000. Cost-analysis for a sustainable nutrient management of fast growing-tree plantations in East-Kalimantan, Indonesia. Forest Ecology and Management 131: 239-253.

Madgwick HAI, P Beets, S Gallagher. 1981. Dry matter accumulation, nutrient and energy content of the above ground portion of 4-year-old stands of Eucalyptus nitens and E. fastigata. New Zealand Journal of Forestry Science 11(1): 53-59.

Minte A. 2004. Funciones de biomasa y volumen para Eucalyptus nitens de cinco años de edad. Tesis Ingeniero Forestal. Valdivia, Chile. Facultad de Ciencias Forestales, Universidad Austral de Chile. 42 p.

Misra RK, CRA Turnbull, RN Cromer, AK Gibbons, AV LaSala. 1998a. Below- and above-ground growth of Eucalyptus nitens in a young plantation. I. Biomass. Forest Ecology and Management 106: 283-293.

Misra RK, CRA Turnbull, RN Cromer, AK Gibbons, AV LaSala, LM Ballard. 1998b. Below- and above-ground growth of Eucalyptus nitens in a young plantation. II. Nitrogen and phosphorus. Forest Ecology and Management 106: 295-306.

Muñoz F. 2002. Balance nutritivo de una plantación de Eucalyptus nitens (Deane et Maiden) Maiden de 7 años de edad en suelos ñadi. Tesis Ingeniero Forestal. Valdivia, Chile. Facultad de Ciencias Forestales, Universidad Austral de Chile. 108 p.

Nyland RD. 2002. Silviculture. Concepts and Applications. Second Edition. New York, USA. McGraw-Hill. 682 p.
Ouro G, P Pérez-Batallón, A Merino. 2001. Effects of sylvicultural practices on nutrient status in a Pinus radiata plantation: Nutrient export by tree removal and nutrient dynamics in decomposing logging residues. Ann. For. Sci. 58: 411-422.

Raison RJ, WJB Crane. 1986. Nutritional costs of shortened rotations in plantation forestry. In Gessel SP. ed. Forest site and productivity. Dordrecht, The Netherlands. Martinus Nijhoff. p. 117-125.

Ringrose C, WA Neilsen. 2005. Growth response of Eucalyptus regnans and soil changes following periodic fertilization. Soil Sci. Soc. Am. J. 69: 1806-1812.

Rodríguez J. 1993. Manual de fertilización. Santiago, Chile. Colección en Agricultura. Pontificia Universidad Católica de Chile. 362 p.

Sankaran KV, TS Grove, S Kumaraswamy, VS Manju, DS Mendham, A M O'Connell. 2005. Export of Nutrients in Plant Biomass Following Harvest of Eucalypt Plantations in Kerala, India. Journal of Sustainable Forestry 20(3): 15-36.

Santana RC, NF De Barros, JCL Neves. 2002. Eficiência de utilização de nutrientes e sustentabilidade da produção em procedências de Eucalyptus grandis e Eucalyptus saligna em sítios florestais do Estado de São Paulo. R. Árvore (Viçosa-MG) 26 (4): 447-457.

Schlatter JE, V Gerding, MI Oñate. 1998. Características y variabilidad de sitios con plantaciones adultas de Pinus radiata D. Don en suelos graníticos de las Regiones VIII y IX. Bosque 19(1): 37-59.

Schumacher MV y MVW Caldeira. 2001. Biomass estimation and nutrient content of a Eucalyptus globulus (Labillardiere) subspecie maidenii plantation. Ciência Florestal (Santa Maria) 11(1): 45-53.

Smethurst PJ, AM Herbert, LM Ballard. 2001. Fertilization effects on soil solution chemistry in three Eucalypt plantations. Soil Sci. Soc. Am. J. 65: 795-804.

Stape JL, D Binkley, MG Ryan. 2004. Eucalyptus production and the supply, use and efficiency of use of water, light and nitrogen across a geographic gradient in Brazil. Forest Ecology and Management 193: 17-31.

Suárez DF, PC Bonomelli. 2001. Evolución de la demanda de N, $\mathrm{P}, \mathrm{K}$ en los tres primeros años de crecimiento de Eucalyptus nitens Maiden y E. globulus Labill en tres ecosistemas de la VIII Región de Chile. In Proceedings of Simposio IUFRO, Valdivia, Chile. 3-15 Septiembre.

Yamada M, T Toma, M Hiratsuka, Y Morikawa. 2004. Biomass and potential nutrient removal by harvesting in short-rotation plantations. In Proceedings of Workshops "Site Management and Productivity in Tropical Plantation Forests". Congo, July 2001 and China, February 2003. Edited by Nambiar EKS, J Ranger, A Tiarks, T Toma. CIFOR 2004. 13 p.

Yost RS, DS DeBell, CD Whitesell, SC Miyasaka. 1988. Early growth and nutrient status of Eucalyptus saligna as affected by nitrogen and phosphorus fertilization. Australian Forestry Research 17: 203-214. 